\title{
Erlotinib plus bevacizumab for EGFR-mutant advanced non-squamous non-small-cell lung cancer patients: ready for first-line?
}

\author{
Helen Adderley ${ }^{1}$, Christoph Jakob Ackermann ${ }^{1}$, Raffaele Califano ${ }^{1,2,3}$ \\ ${ }^{1}$ Department of Medical Oncology, The Christie NHS Foundation Trust, Manchester, UK; ${ }^{2}$ Department of Medical Oncology, Manchester \\ University NHS Foundation Trust, Manchester, UK; ${ }^{3}$ Division of Cancer Sciences, University of Manchester, Manchester, UK \\ Correspondence to: Dr. Raffaele Califano. The Christie NHS Foundation Trust, Manchester, M20 4BX, UK. Email: Raffaele.Califano@christie.nhs.uk. \\ Provenance: This is an invited article commissioned by the Section Editor Dr. Song Xu (Department of Lung Cancer Surgery, Tianjin Medical \\ University General Hospital; Tianjin Key Laboratory of Lung Cancer Metastasis and Tumor Microenvironment, Lung Cancer Institute, Tianjin, \\ China). \\ Comment on: Saito H, Fukuhara T, Furuya N, et al. Erlotinib plus bevacizumab versus erlotinib alone in patients with EGFR-positive advanced non- \\ squamous non-small-cell lung cancer (NEJ026): interim analysis of an open-label, randomised, multicentre, phase 3 trial. Lancet Oncol 2019;20:625-35.
}

Submitted Sep 04, 2019. Accepted for publication Sep 17, 2019.

doi: 10.21037/atm.2019.09.116

View this article at: http://dx.doi.org/10.21037/atm.2019.09.116

EGFR tyrosine kinase inhibitors (TKIs) represent the standard of care for first-line treatment for patients with advanced EGFR mutant non-small cell lung cancer (NSCLC) with superiority in overall response rate (ORR), progression free survival (PFS) and quality of life compared to platinum-based chemotherapy (ChT) (1).

The incidence of EGFR activating mutation varies from $10-15 \%$ for Caucasian to up to $35-50 \%$ in East Asians patients. A never or light smoking history, adenocarcinoma histology and female sex are associated with a higher mutation incidence (2,3). First-line EGFR-TKIs achieve ORR of $60-80 \%$ but ultimately all patients develop progressive disease. In almost two thirds of patients an acquired amino acid substitution at position 790 (T790M) of the EGFR exon 20 domain is the underlying mechanism of resistance conferring to reduced ATP competitive TKIbinding and loss of drug activity (4). Osimertinib, a third generation TKI, has proven to overcome T790M-induced resistance with improved PFS and ORR compared to ChT [PFS: 10.1 vs. 4.4 months, hazard ratio (HR) $0.3, \mathrm{P}<0.001$, ORR: $71 \%$ vs. $31 \%$, odds ratio (OR) $5.39, \mathrm{P}<0.001]$ (5) and has been licensed by the Food and Drug Administration (FDA) and European Medicine Agency (EMA) in the firstline setting due to improved PFS (18.9 vs. 10.2 months, HR $0.46, \mathrm{P}<0.001)$ and tolerability compared to the firstgeneration TKIs erlotinib and gefitinib (6).
The benefit of anti-PD-(L)1 checkpoint inhibitor (CPI) monotherapy in EGFR-mutant patients, even with tumors harboring a PD-L1 expression of $\geq 50 \%$, is questionable and current licenses for the use of first-line CPI exclude patients with an EGFR sensitizing mutation or $A L K$ gene rearrangement $(7,8)$.

At progression after EGFR-TKI treatment, prognosis is very poor and salvage ChT shows only limited benefit. Therefore, combination therapy to improve first line outcomes is an attractive option to explore.

The combination of erlotinib with bevacizumab has been investigated in two previous phase II clinical trials: BELIEF (9) and JO25567 (10). Bevacizumab, an anti-angiogenic monoclonal antibody, targets the VEGF signalling pathway and has been shown to prolong survival when combined with first-line platinum-based ChT in non-squamous NSCLC (11-13). In the BELIEF study (9), 109 patients were randomised to erlotinib plus bevacizumab vs. erlotinib alone based on stratification according to the presence of pretreatment T790M mutation. Results demonstrated that the 37 patients with baseline T790M mutation had a longer PFS than those without: 16.0 months (12.7 to not estimable) $v s$. 10.5 months (9.4-14.2 months). In the JO25567 study (10), 154 patients were randomly assigned to combination therapy $v s$. erlotinib alone and were excluded if they had T790M mutation at baseline. Again, PFS was longer in the 
erlotinib and bevacizumab arm (16.0 vs. 9.7 months, HR 0.54, 95\% CI: 0.36-0.79; P=0.0015). Notably, the JO25567 study was not powered to assess overall survival (OS).

Contrary to these positive trials, results of a phase II randomised study evaluating erlotinib plus bevacizumab $v s$. erlotinib were recently published in fAMA Oncology (14). Eighty-eight patients were randomly assigned (1:1) to combination therapy $v s$. erlotinib. There was no improvement in PFS (17.9 vs. 13.5 months, HR 0.81; 95\% CI: $0.50-1.31 ; \mathrm{P}=0.39$ ) or OS (32.4 vs. 50.6 months, HR 1.41; $95 \%$ CI: $0.71-2.81 ; \mathrm{P}=0.33$ ) for combination therapy and erlotinib arm, respectively. Study limitations include patient selection ( $85 \%$ were white), and the lack of blinded independent radiology review. OS data from this study should be interpreted with caution due to small number of events and limited access to subsequent therapies, which hindered analysis of post study therapies on OS results.

The interim analysis of NEJ026 was recently reported in The Lancet Oncology (15). In this phase III randomised, multicentre, open label, study across 69 centres in Japan, participants were assigned to receive erlotinib $150 \mathrm{mg}$ OD with bevacizumab $15 \mathrm{mg} / \mathrm{kg}$ once every 21 days $v s$. erlotinib alone. Patients with asymptomatic brain metastasis were eligible for enrolment whilst patients whose tumours harboured T790M mutations at baseline or who had received previous ChT for advanced stage disease were excluded. Two hundred and twenty-eight patients were enrolled and randomly assigned. This interim analysis was performed at data cut off when 117 PFS (primary endpoint) events had occurred.

The baseline characteristics of both treatment groups were well balanced with similar incidence $(32 \%)$ of brain metastasis in each treatment arm. With a median follow up of 12.4 months, median PFS was longer in the erlotinib plus bevacizumab group (16.9 vs. 13.3 months, HR 0.605, 95\% CI: $0.417-0.878 ; \mathrm{P}=0.016)$. In the post hoc subgroup analysis, erlotinib plus bevacizumab was superior in most subgroups, although this was not statistically significant. Median PFS was longer in those with Leu858Arg mutations in the combination group (17.4 vs. 13.7 months, HR 0.57, 95\% CI: 0.33-0.97) however no difference was found in those with exon 19 deletions (16.6 vs. 12.4 months, HR 0.69, 95\% CI: 0.41-1.16). Patients without CNS metastasis had improved PFS with combination erlotinib and bevacizumab (HR 0.56, 95\% CI: 0.35-0.90) with no significant difference identified in those with CNS involvement (HR 0.78, 95\% CI: $0.42-1.43)$.

Grade $\geq 3$ toxicity was reported in $88 \%(98 / 112)$ of patients in the combination group and 46\% (53/114) of patients in the erlotinib only group. The most common grade 3-4 adverse event was rash, $21 \%$ in both treatment arms, and serious adverse events occurred in $8 \% v s$. $4 \%$ of patients enrolled in the combination group and erlotinib group, respectively. Twenty-nine percent (33/112) patients discontinued bevacizumab due to adverse events.

Study limitations include a small sample size and lack of power to assess PFS in subgroup analysis. Furthermore, the proportion of patients with ECOG PS of 0 was high $(59 \%)$ as was the proportion of patients with post-operative recurrence $(19 \%)$. These, combined with exclusion of patients harbouring de novo T790M mutation, may have led to longer PFS than reported in previous trials.

Notably, the NEJ026 trial was conducted to evaluate the impact on OS from erlotinib and bevacizumab combination as follow on from J025567 study, yet PFS was used as primary outcome.

Results of NEJ026 (15) and sequencing of therapy does need to be considered alongside evidence from recent clinical trials including FLAURA (6), NEJ009 (16) and IMpower-150 (17). FLAURA demonstrated that first-line Osimertinib achieved a longer PFS (18.9 vs. 10.2 months, HR 0.46; 95\% CI: $0.37-0.57$; $\mathrm{P}<0.001)$ vs. erlotinib or gefitinib, regardless of baseline T790M status. Adverse events of grade 3 or higher were lower with osimertinib than standard TKI therapy (34\% vs. 45\%). OS (secondary endpoint) data is still immature (6). The NEJ009 evaluated combination gefitinib plus platinum doublet ChT vs. gefitinib alone demonstrating longer PFS for the combination (20.9 vs. 11.2 months, HR 0.493; 95\% CI: $0.39-0.62 \mathrm{P}<0.001)$ but no difference in PFS2 (16). IMpower-150 evaluated first-line carboplatin/paclitaxel/ bevacizumab + atezolizumab (ABCP) vs. carboplatin/ paclitaxel + bevacizumab (BCP). Patients with a sensitizing EGFR mutation were eligible, after TKI failure. In patients with sensitising EGFR mutations treated with $\mathrm{ABCP}(\mathrm{n}=26)$ there was a longer OS (NE vs. 17.5 months, HR 0.31; 95\% CI: 0.11-0.83) than patients who received BCP $(n=32)$. Notably, in patients with EGFR sensitising mutations who previously received an EGFR-TKI $(n=50)$, the only population to be allowed in the study as per protocol, there was a trend towards longer OS which was not statistically significance [NE vs. 17.5 months, HR 0.39 (0.14-1.07)] (17). This subgroup analysis needs to be interpreted with caution due to small sample sizes and confirmatory studies are needed.

With an increasing number of therapies being 
investigated in EGFR mutant NSCLC the landscape of firstand later-line of therapy is becoming increasingly complex. To improve patient outcomes, it is of utmost importance to understand the mechanisms of TKI resistance such as upregulation of MET, HGF, HER2 mutations, HER3 overexpression, activation of IGF-1R or downregulation of PTEN (18). Furthermore, it is crucial to develop reliable biomarkers to early expose acquired TKI resistance.

With the caveat of cross-trial comparison, the PFS reported in NEJ026 (15) seems to be inferior to what reported in FLAURA (6) and it would be very important to see the updated OS data from FLAURA, when available. Without demonstrable OS improvement, PFS could be seen as an inadequate measure of benefit for the combination of two known active drugs. Until NEJ026 OS data is mature, we anticipate that the combination of erlotinib and bevacizumab will not be considered a standard first line option. Combination EGFR-TKI and bevacizumab therapy may have a role when access to osimertinib in the first line setting is limited but with increased toxicity and associated costs. Two phase II clinical trials evaluating the combination of osimertinib and bevacizumab in the $1^{\text {st }}$ line setting (NCT02803203) (19) and after progression on an EGFRTKI other than a $3^{\text {rd }}$ generation TKI (20) are ongoing. The results of these studies may help shed more light on the most appropriate treatment strategies in EGFR mutant patients.

\section{Acknowledgments}

None.

\section{Footnote}

Conflicts of Interest: R Califano has received honoraria for consultancy and advisory board from Astrazeneca, Roche, Novartis and Boheringer Ingelheim. The other authors have no conflicts of interest to declare.

Ethical Statement: The authors are accountable for all aspects of the work in ensuring that questions related to the accuracy or integrity of any part of the work are appropriately investigated and resolved.

\section{References}

1. Hanna N, Johnson D, Temin S, et al. Systemic Therapy for Stage IV Non-Small-Cell Lung Cancer: American
Society of Clinical Oncology Clinical Practice Guideline Update Summary. J Oncol Pract 2017;13:832-7.

2. Dogan S, Shen R, Ang DC, et al. Molecular epidemiology of EGFR and KRAS mutations in 3,026 lung adenocarcinomas: higher susceptibility of women to smoking-related KRAS-mutant cancers. Clin Cancer Res 2012;18:6169-77.

3. Shi Y, Au JS, Thongprasert S, et al. A prospective, molecular epidemiology study of EGFR mutations in Asian patients with advanced non-small-cell lung cancer of adenocarcinoma histology (PIONEER). J Thorac Oncol 2014;9:154-62.

4. Ma C, Wei S, Song Y. T790M and acquired resistance of EGFR TKI: a literature review of clinical reports. J Thorac Dis 2011;3:10-8.

5. Mok TS, Wu YL, Ahn MJ, et al. Osimertinib or PlatinumPemetrexed in EGFR T790M-Positive Lung Cancer. N Engl J Med 2017;376:629-40.

6. Soria JC, Ohe Y, Vansteenkiste J, et al. Osimertinib in Untreated EGFR-Mutated Advanced Non-Small-Cell Lung Cancer. N Engl J Med 2018;378:113-25.

7. Lisberg A, Cummings A, Goldman JW, et al. A Phase II Study of Pembrolizumab in EGFR-Mutant, PD-L1+, Tyrosine Kinase Inhibitor Naive Patients With Advanced NSCLC. J Thorac Oncol 2018;13:1138-45.

8. Lee CK, Man J, Lord S, et al. Checkpoint Inhibitors in Metastatic EGFR-Mutated Non-Small Cell Lung Cancer-A Meta-Analysis. J Thorac Oncol 2017;12:403-7.

9. Rosell R, Daphni U, Felip E, at al. Erlotinib and bevacizumab in patients with advanced non-small-cell lung cancer and activating EGFR mutations (BELIEF): an international, multicentre, single-arm, phase 2 trial. Lancet Respir Med 2017;5:435-44.

10. Seto T, Kato T, Nishio M, et al. Erlotinib alone or with bevacizumab as fi rst-line therapy in patients with advanced non-squamous non-small-cell lung cancer harbouring EGFR mutations (JO25567): an open-label, randomised, multicentre, phase 2 study. Lancet Oncol 2014;15:1236-44.

11. Sandler A, Gray R, Perry MC, et al. Paclitaxel-carboplatin alone or with bevacizumab for non-small-cell lung cancer. N Engl J Med 2006;355:2542-50.

12. Reck M, von Pawel J, Zatloukal P, et al. Phase III trial of cisplatin plus gemcitabine with either placebo or bevacizumab as fi rst-line therapy for nonsquamous non-small-cell lung cancer: AVAiL. J Clin Oncol 2009;27:1227-34.

13. Niho S, Kunitoh H, Nokihara H, et al, for the JO19907 
Study Group. Randomized phase II study of first-line carboplatin-paclitaxel with or without bevacizumab in Japanese patients with advanced non-squamous non-smallcell lung cancer. Lung Cancer 2012;76:362-7.

14. Stinchcombe TE, Jänne PA, Wang X, et al. Effect of Erlotinib Plus Bevacizumab vs Erlotinib Alone on Progression-Free Survival in Patients With Advanced EGFR-Mutant Non-Small Cell Lung Cancer: A Phase 2 Randomized Clinical Trial. JAMA Oncol 2019. [Epub ahead of print].

15. Saito H, Fukuhara T, Furuya N, et al. Erlotinib plus bevacizumab versus erlotinib alone in patients with EGFR-positive advanced non-squamous non-small-cell lung cancer (NEJ026): interim analysis of an open-label, randomised, multicentre, phase 3 trial. Lancet Oncol 2019;20:625-35.

16. Nakamura A, Inoue A, Morita S, et al. Phase III study comparing gefitinib monotherapy $(\mathrm{G})$ to combination therapy with gefitinib, carboplatin, and pemetrexed (GCP) for untreated patients (pts) with advanced non-small cell

Cite this article as: Adderley H, Ackermann CJ, Califano R. Erlotinib plus bevacizumab for EGFR-mutant advanced nonsquamous non-small-cell lung cancer patients: ready for firstline? Ann Transl Med 2019;7(Suppl 8):S346. doi: 10.21037/ atm.2019.09.116 lung cancer (NSCLC) with EGFR mutations (NEJ009). J Clin Oncol 2018;36:9005.

17. Reck M, Mok TSK, Nishio M, et al. Atezolizumab plus bevacizumab and chemotherapy in non-small-cell lung cancer (IMpower150): key subgroup analyses of patients with EGFR mutations or baseline liver metastases in a randomised, open-label phase 3 trial. Lancet Respir Med 2019;7:387-401.

18. Lin Y, Wang X, Jin H. EGFR-TKI resistance in NSCLC patients: mechanisms and strategies. Am J Cancer Res 2014;4:411-35.

19. Yu HA, Kim R, Makhnin A, et al. A phase 1/2 study of osimertinib and bevacizumab as initial treatment for patients with metastatic EGFR-mutant lung cancers. J Clin Oncol 2019;37:9086.

20. Akamatsu H, Teraoka S, Morita S, et al. Phase I/II Study of Osimertinib With Bevacizumab in EGFR-mutated, T790M-positive Patients With Progressed EGFR-TKIs: West Japan Oncology Group 8715L (WJOG8715L). Clin Lung Cancer 2019;20:e492-4. 